\title{
AGE OF HIV ACQUISITION AFFECTS THE RISK OF MULTI-MORBIDITY AFTER 25 YEARS OF INFECTION EXPOSURE
}

\author{
G. GUARALDI ${ }^{1}$, A. MALAGOLI ${ }^{1}$, J. MILIC ${ }^{1,5}$, I. PINTASSILGO $^{4}$, E. ROSSI ${ }^{3}$, N. RIVA ${ }^{1}$, I. FRANCONI $^{1}$, \\ A. SANTORO, P. SORIN ${ }^{2}$, A. STREINU-CERCEL ${ }^{2}$, M. DE ROSA $^{3}$, C. MUSSINI ${ }^{1}$
}

1. Department of Infectious Diseases, Modena HIV Metabolic Clinic, University of Modena and Reggio Emilia, Modena, Italy; 2. National Institute for Infectious Diseases "Prof. Dr.

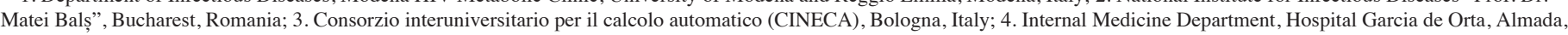
Portugal; 5. Clinical and Experimental Medicine PhD Program, University of Modena and Reggio Emilia, Modena, Italy.

Corresponding author: Giovanni Guaraldi, Universita degli Studi di Modena e Reggio Emilia, Italy, giovanni.guaraldi@unimore.it

\begin{abstract}
Introduction: Understanding the intersection of HIV, aging and health is crucial due to the increasing number of people aging with HIV. Objective: The objective of the study was to assess the prevalence of, and risk factors for individual comorbidities and multi-morbidity in people living with HIV with similar duration of HIV infection, notwithstanding a 25-year difference at the time of HIV acquisition. Methods: In a crosssectional multicentre retrospective study, we compared three match-control age groups. The "Young" were selected from Romania and included HIV-positive patients prenatally infected and assessed at the age of 25-30 years. The "Old" and the "Geriatric" were selected from Italy. These respectively included subjects infected with HIV at the age of 25 years and assessed at the age of 50-55 years, and those infected at the age of 50 years and assessed at the age of 75-80 years. Each group was sex and age matched in a 1:5 ratio with controls selected from the CINECA ARNO database from Italy. We described non-infectious comorbidities (NICM), including cardiovascular disease, hypertension, dyslipidaemia, diabetes, chronic kidney disease, and multi-morbidity $(\mathrm{MM} \geq 3 \mathrm{NICM})$. Results: MM prevalence in the "Young" group compared to controls was $6.2 \%$ vs $0 \%$, while in the "Geriatric" was " $68.2 \%$ vs 3.6\%. Using "Young" as a reference, in multivariate analyses, predictors for MM were as follows: HIV serostatus $(\mathrm{OR}=47.75$, IQR 14.78-154.25, p<0.01) and "Geriatric" vs "Young" $(\mathrm{OR}=30.32$, IQR 5.89-155.98, p<0.01). Conclusion: These data suggest that age at acquisition of HIV should be considered as a risk factor for NICM and MM.
\end{abstract}

Key words: HIV, age, multi-morbidity, acquisition.

J Frailty Aging 2019;8(2)88-92

Published online March 22, 2019, http:/ / dx.doi.org/10.14283/jfa.2019.6

\section{Introduction}

The improved understanding of the intersection of HIV, aging and health is an urgent issue due to the increasing number of people aging with HIV as the synergistic result of two concurrent phenomena: people living with HIV (PLWH) live longer and more people catch HIV at an older age $(1,2)$. The former fact results from the increased life expectancy guaranteed by antiretroviral therapy (ART); the latter from a changed perception of sexual risk in older people $(3,4)$.

The aging epidemic of PLWH arouses potential concern due to physical, neurocognitive and psychological issues that can accompany both aging and HIV infection (5).

Aging with HIV is often linked to non-infectious comorbidities (NICM), including cardiovascular disease (CVD), hypertension (HTN), dyslipidaemia (DLP), type 2 diabetes mellitus (T2DM), chronic kidney disease (CKD), osteopenia/osteoporosis (O/O), and non-AIDS cancers (NAC). These heterogeneous NICM share age and HIV infection as independent risk factors and tend to aggregate into complex multi-morbidity (MM) patterns $(6,7)$.

The current literature focusing on HIV in older people concentrates on studies comparing older with younger HIVpositive individuals, or else comparing HIV-positive to HIV- negative older individuals $(8,9)$. Such comparative studies have begun to highlight the diversity of individuals aging with HIV, in terms of behavioural factors, social vulnerability, and ethnoracial disparities, which might contribute to different patterns of aging (1).

In a published study including ART-experienced patients, we showed that host factors and duration of HIV infection were associated with an increased risk of MM compared to the general population (10).

Comparing PLWH with diverse age but similar exposure to HIV might help us to better depict the relationship between age and HIV. We hypothesized that age of HIV acquisition impacts on the prevalence of NICM.

The objective of the study was to assess the prevalence of and risk factors for individual comorbidities and multimorbidity in PLWH with similar duration of HIV infection, notwithstanding a 25-year difference at the time of HIV acquisition.

In detail, we compared PLWH for 25 years who acquired $\mathrm{HIV}$ infection at birth, at 25 and at 50 years of age with age and sex matched community dwelling people aged 25, 50 and 75 years. 


\section{THE JOURNAL OF FRAILTY \& AGING}

\section{Materials and methods}

This is a match-control study, including ART-experienced patients from:

1. Modena HIV Metabolic Clinic (MHMC), a tertiary level HIV clinic opened in 2003. Collecting over 4,500 HIV patients referred from all over Italy, MHMC provides annual multidisciplinary evaluation to PLWH, including assessment for metabolic alterations, NICM and MM.

2. Romanian National Institute for Infectious Diseases "Prof. Dr. Matei Bals" (Romanian HIV Clinic, RHC), which was established in 1990 and gathered over 1,094 cases of paediatric HIV.

Patients selected from these two centres were included on a number of conditions: first of all, if they had been living with HIV for 25 years and within the three pre-specified age groups; secondly, if they were currently undergoing ART with undetectable plasma HIV RNA viral load; finally, if they had been screened for NICM.

These patients were age and sex matched with individuals from the general population selected from Consorzio interuniversitario per il calcolo automatico (CINECA ARNO) observatory, in Italy.

3. The CINECA ARNO Observatory is an on-line, multicentre observational database in which population-based data are collected and epidemiological methods are used to combine and aggregate large volumes of health and healthcare-related files for each participant (11). These data include primary care provider-generated medication prescriptions, inpatient hospital records and discharge, diagnostic laboratory tests and radiographic examinations. From this administrative cohort, NICM diagnoses are provided by International Classification of Diseases, Tenth Revision. CINECA ARNO does not collect lifestyle, anthropometric and metabolic data.

Data were collected in the fourth quarter of 2016.

The following age group strata were identified:

- Group "Young" was selected from RHC. It comprises patients prenatally infected and assessed at the age of 25-30 years;

- Group "Old" was selected from MHMC. It comprises patients infected at the age of 25 years and assessed at the age of 50-55 years;

- Group "Geriatric" was selected from MHMC. It comprises patients diagnosed at the age of 50 years and assessed at the age of 75-80 years;

- Controls were selected from the CINECA ARNO observational database and matched according to age, sex, and race.

The matching could not compensate for relevant sociodemographic differences between MHMC and RHC, while both of them were compared with general Italian population collected by CINECA ARNO.

HIV variables included current and nadir CD4 cell counts, current and cumulative exposure to ART drug classes.

Study outcomes comprised the following NICM diagnosed according to guideline-based criteria: CVD, HTN, T2DM, lipodystrophy (12).

MM was defined as the concurrent presence of $\geq 3 \mathrm{NICM}$ $(13,14)$.

\section{Statistical analyses}

Comparisons between groups were performed using $\mathrm{x}^{2}$ test for categorical variables with Bonferroni adjusted post-hoc analyses (significant level set at $\mathrm{p}<0.017$ ) and t-test or MannWhitney U-test for normally and non-normally distributed continuous variables, respectively.

A logistic regression analyses was performed to identify the probability of MM comparing both "Old" and "Geriatric" groups to "Young" used as a reference, and by comparing HIV patients to the general population after correction for sex.

A 1:5 matching of HIV patients with CINECA-ARNO records allowed us to obtain medium Cohen's effect size as 0.6, enabling us to estimate a power size of 0.87 .

Statistical analyses were performed with $\mathrm{R}$ software version 3.4.2 for Linux.

\section{Results}

A total number of 131 HIV positive patients (the "Young" group involved 32 patients, while "Old" and "Geriatric" contained 77 and 22 patients respectively) and 655 controls (the "Young" group comprised 160 patients, while "Old" and "Geriatric" contained 385 and 110 patients respectively) were included.

Univariate analysis was conducted to describe demographic and anthropometric characteristics, HIV variables, cumulative

Figure 1

Prevalence of comorbidities and MM in the study groups compared to the general population

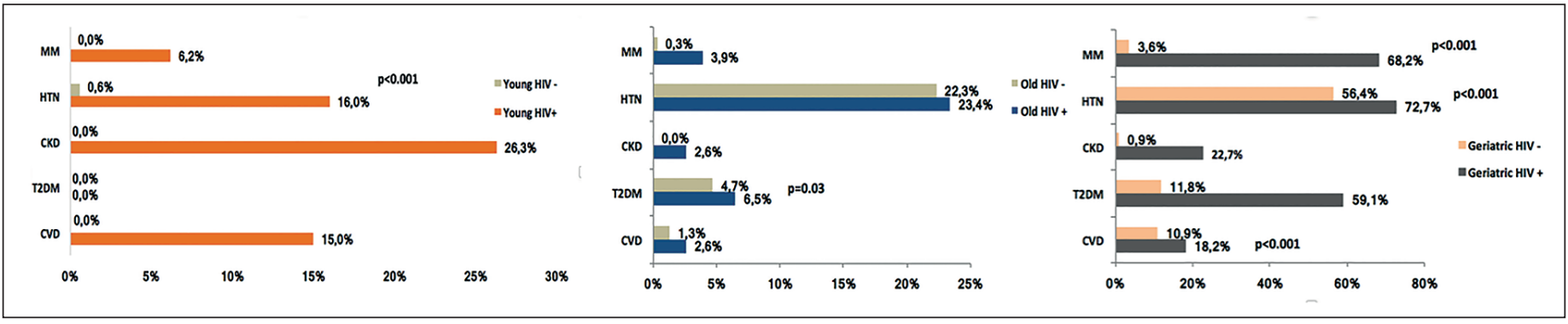


Table 1

Demographic and HIV-related variables in HIV study groups

\begin{tabular}{|c|c|c|c|c|c|}
\hline & Total & Group A (Young) & Group B (Old) & Group C (Geriatric) & p-value \\
\hline Number $(\%)$ & $131(100 \%)$ & $32(24.43 \%)$ & $77(58.78 \%)$ & $22(16.79 \%)$ & \\
\hline \multicolumn{6}{|c|}{ Demographic and anthropometric characteristics } \\
\hline BMI, $\mathrm{kg} / \mathrm{m}^{2}$, mean $( \pm \mathrm{SD})$ & $23.84(3.89)$ & $23.58(3.96)$ & $23.49(4)$ & $25.34(3.15)$ & 0.14 \\
\hline Smoker, n (\%) & $39(31.71 \%)$ & $5(16.67 \%)$ & $33(46.48 \%)$ & $1(4.55 \%)$ & $<0.01$ \\
\hline IVDU, n (\%) & $44(35.2 \%)$ & $0(0 \%)$ & $44(57.14 \%)$ & $0(0 \%)$ & $<0.01$ \\
\hline $\mathrm{HBV}, \mathrm{n}(\%)$ & $13(12.5 \%)$ & $8(33.33 \%)$ & $5(7.94 \%)$ & $0(0 \%)$ & $<0.01$ \\
\hline $\mathrm{HCV}, \mathrm{n}(\%)$ & $42(40.38 \%)$ & $1(4.17 \%)$ & $40(63.49 \%)$ & $1(5.88 \%)$ & $<0.01$ \\
\hline \multicolumn{6}{|l|}{ HIV variables } \\
\hline AIDS diagnosis, $\mathrm{n}(\%)$ & $42(38.18 \%)$ & $17(85 \%)$ & $18(26.47 \%)$ & $7(31.82 \%)$ & $<0.01$ \\
\hline \multicolumn{6}{|l|}{ ART (cumulative exposure) } \\
\hline NRTI, months, median (IQR) & $133(82.75-185.5)$ & $172.5(121.5-222.5)$ & $119(59.25-181.25)$ & $129(99.25-167.75)$ & $<0.01$ \\
\hline PI, months, median (IQR) & $80.5(37-143.25)$ & $154.5(69.25-204.25)$ & $72(34-120)$ & $47(23.91)$ & $<0.01$ \\
\hline NNRTI, months, median (IQR) & $46.5(16.5-95.75)$ & $42(16.5-98.5)$ & $46(16-94)$ & $73.5(33.75-105.5)$ & 0.59 \\
\hline INSTI, months, median (IQR) & $32(17-51.5)$ & $43(27.75-63.75)$ & $22(14-50)$ & $19(14.25-25.25)$ & 0.23 \\
\hline \multicolumn{6}{|l|}{ Biochemical parameters } \\
\hline Hemoglobin, g/dL, mean $( \pm$ SD $)$ & $14.42(1.81)$ & $14.35(1.69)$ & $14.33(1.94)$ & $14.84(1.58)$ & 0.45 \\
\hline Platelets, $10^{9} / \mathrm{L}$, mean $( \pm$ SD) & $191.13(59.94)$ & $207.81(51.08)$ & $181.82(66.71)$ & $190.94(46.62)$ & 0.15 \\
\hline $\mathrm{WBC}, 10^{12} / \mathrm{L},( \pm \mathrm{SD})$ & $4337.48(3140.39)$ & $6344.69(1567.48)$ & $3679(3281.11)$ & $2805.76(3178.12)$ & $<0.01$ \\
\hline Triglycerides, mg/dL, mean $( \pm \mathrm{SD})$ & $161.79(87.14)$ & $149.95(88.24)$ & $155.3(85.05)$ & $193.73(89.27)$ & 0.06 \\
\hline $\mathrm{HbA} 1 \mathrm{C}, \%$, median (IQR) & $5.5(5.2-6.2)$ & NA & $5.4(5.0-5.7)$ & $6.15(5.57-7.52)$ & 0.005 \\
\hline \multicolumn{6}{|l|}{ NICM (study outcomes) } \\
\hline CKD, n (\%) & $12(10.17 \%)$ & $5(26.32 \%)$ & $2(2.6 \%)$ & $5(22.73 \%)$ & $<0.01$ \\
\hline CVD, n (\%) & $9(7.56 \%)$ & $3(15 \%)$ & $2(2.6 \%)$ & $4(18.18 \%)$ & 0.02 \\
\hline Hypertension, n (\%) & $38(30.65 \%)$ & $4(16 \%)$ & $18(23.38 \%)$ & $16(72.73 \%)$ & $<0.01$ \\
\hline Lipodystrophy, n (\%) & $87(69.6 \%)$ & $20(76.92 \%)$ & $46(59.74 \%)$ & $21(95.45 \%)$ & $<0.01$ \\
\hline Diabetes, n (\%) & $18(14.29 \%)$ & $0(0 \%)$ & $5(6.49 \%)$ & $13(59.09 \%)$ & $<0.01$ \\
\hline
\end{tabular}

Abbreviations: SD - standard deviation; BMI - body mass index; HIV - human immunodeficiency virus; IQR - interquartile range; IVDU - intravenous drug user; HBV - hepatitis B virus; HCV - hepatitis C virus; NRTI - nucleoside reverse transcriptase inhibitor; NNRTI - non-nucleoside reverse transcriptase inhibitor; PI - protease inhibitor; INSTI - integrase strand transfer inhibitors; WBC - white blood cell; GOT -glutamic oxaloacetic transaminase; GPT - glutamate-pyruvate transaminase; HTN - hypertension; CVD - cardiovascular disease; CKD - chronic kidney disease 


\section{THE JOURNAL OF FRAILTY \& AGING}

exposure to ART, biochemical parameters and study outcomes in HIV positive groups. With regard to comorbidities, all NICM were more prevalent in the "Geriatric" group (table 1).

The comparison between HIV groups and controls is depicted in Figure 1, which shows that the prevalence of NICM is higher in PLWH.

A multivariable linear regression model was built to identify predictors of MM in all examined groups, using the "Young" as reference. HIV serostatus was the highest risk factor for MM (OR=47.75, IQR 14.78-154.25, p<0.01). Higher risk for MM was displayed in "Geriatric" vs "Young" (OR=30.32, IQR 5.89-155.98, $\mathrm{p}<0.01)$, but not comparing "Young" vs "Old" $(\mathrm{OR}=0.81$, IQR $0.14-4.65, \mathrm{p}=0.817)$ and the female gender $(\mathrm{OR}=0.68, \mathrm{IQR} 0.18-2.53, \mathrm{p}=0.561)$.

\section{Discussion}

This study depicted NICM and MM in PLWH over 25 years. As expected, the prevalence of these conditions was much higher in HIV-positive patients, when compared to CINECA records.

The high burden of MM affecting PLWH was shown for the first time seven years ago at MHMC (6), and it has been reproduced in several other HIV cohorts around the globe since then (7). At the time, it was observed that NICM and MM were age-related conditions (15). This finding ignited a longstanding debate regarding accentuated or accelerated aging in PLWH. More importantly, it introduced the idea that aging in HIV is not simply the effect of anagraphical age. We have previously demonstrated that HIV duration is more important than anagraphical age for the risk of NICM and MM (10).

In the present study, we wanted to show that regardless of the same, long duration of HIV (25 years), age of HIV acquisition impacts on age-related conditions. Apparently, our data suggest a "U-curve" phenomenon in which HIV acquisition at birth entailed a higher risk for NICM and MM than in people who acquired HIV as young adults (25-30 years old). Patients who acquired HIV at the age of 50 years or more displayed the highest prevalence of NICM (except CKD) and MM. This age category was observed to be an independent risk factor for MM, after correction for anagraphical age. On the contrary, age of HIV acquisition did not affect immunological parameters, underlining the high probability of obtaining virologic success in a population reaching undetectable HIV viral load.

This study contributes to the understanding that HIV is an "age modulator", that is a chronic health condition in which not only duration but also age of acquisition modulate the biological age of PLWH. The heightened risk for MM observed in PLWH, when compared to the general population, is therefore the result of three time variables: anagraphical age, HIV duration and age of HIV acquisition.

This study also described three age groups which differed for multiple demographic, HIV and clinical characteristics.
The "Young" belonged to a unique epidemiology setting in which lower socio-economic status and healthcare access difficulties, with limited screening and preventive strategies, may have contributed to the disease burden of the "Young" group (16).

The "Old" were representative of the "first wave" of HIV epidemic in Italy. They were more likely to have acquired HIV with intravenous drug use and as expected, they were more likely to be smokers and heavy drinkers (17).

The "Geriatric" are an emerging population. Over the past fifteen years, a significant increase in new HIV/AIDS diagnoses has been observed in the elderly population (18). This new epidemiological shift has been attributed to a longer sex life, lifestyle and changes in sexual behaviour, poor sexual health education and misconceptions about absence of sexually transmitted disease in later life. In this population, NICM may have been present before HIV acquisition $(19,20)$. In this chronological-age vulnerable population, HIV per se and ART may represent an additional risk factor for NICM and MM.

In the European epidemiological context, where an increasing number of patients acquire HIV at an older age (2), dedicated programs for HIV testing in elderly individuals at risk for HIV and routine screening for NICM in HIV elderly patients should be reinforced. The fact that the "Geriatric" was the most vulnerable group underlines the need of a "geriatric HIV care" in the setting of the HIV epidemic.

Putting this study in the context of contemporary HIV care with immediate access and less toxic ART may hopefully reduce the burden of NICM and MM in future years. In our study, cumulative exposure to "older" drug regimen including NRTI and PI/r differed between groups, while the newer NNRTI and INSTI regimens were similar.

This study has relevant limitations, some of which inherent in the cross-sectional nature of the study. The major limitation lies in the non-obvious reproducibility of these results, particularly with regard to the "Young" group. The collaboration between MCMC and RHC was necessary in order to provide an age group rarely represented in Italy. Italy and Romania have a different healthcare system. Moreover, it is still difficult to have routine assessments for multiple NICM in perinatally infected patients. Additionally, evaluation of the NICM in this population differs from the standard assessment in adults. The burden of NICM identified in this study suggests that routine screening for NICM is a necessary process in the care of PLWH.

The small sample size was also a limitation of this study, related to both the lack of data on NICM in the "Young" from the RHC, and the small number of "Geriatric" from MHMC.

The small sample size may have been responsible for the absence of any patients with cancer.

In conclusion, these data suggest that age at acquisition of HIV infection should be considered as a risk factor for NICM and MM. 


\section{AGE AT HIV ACQUISITION AFFECTS THE RISK OF MULTI-MORBIDITY AFTER 25 YEARS OF INFECTION EXPOSURE}

Funding: Supported in part by a research grant awarded under the Investigator Initiated Study Program of Merck Sharp \& Dohme Corp. The views expressed in this report are from the authors and do not necessarily represent those of Merck Sharp \& Dohme Corp.

Acknowledgments: We are grateful to Dr. Barbara Beghetto, Dr. Giulia Nardini and Dr. Enrica Roncaglia for data management and to Prof. Davide Mazzi for the English language revision.

Conflict of interests: None to declare.

\section{References}

1. Chambers LA, Wilson MG, Rueda $\mathrm{S}$ et al. Evidence Informing the Intersection of HIV, Aging and Health: A Scoping Review. AIDS Behav, 2014; 18 (4): 661-75.

2. Lazarus JV, Nielsen KK. HIV and people 50 years old in Europe. HIV Med, 2010; 11 (7): 479-81.

3. Cooperman NA, Arnsten JH, Klein RS. Current sexual activity and risky sexual behavior in older men with or at risk for HIV infection. AIDS EducPrev2007; 19 (4): 321-33.

4. CDC, Center for Disease Control and Prevention. HIV/ AIDS among Persons Aged 50 and Older. 2008;

5. Önen NF, Overton ET, Seyfried W et al. Aging and HIV infection: a comparison between older HIV-infected persons and the general population. HIV Clin Trials 2010; 11 (2): 100-9.

6. Guaraldi G, Orlando G, Zona S, et al. Premature age-related comorbidities among HIV-infected persons compared with the general population. Clin Infect Dis, 2011; 53 (11): 1120-6.

7. Schouten J, Wit FW, Stolte IG, et al. Cross-sectional comparison of the prevalence of age-associated comorbidities and their risk factors between HIV-infected and uninfected individuals: the AGE IV Cohort Study. Clin Infect Dis, 2014; pii: ciu701

8. Althoff KN, Jacobson LP, Cranston RD, et al. Age, comorbidities, and AIDS predict a frailty phenotype in men who have sex with men. J Gerontol A BiolSci Med Sci, 2014; 69 (2): 189-98.
9. Samji H, Cescon A, Hogg RS, et al.Closing the Gap: Increases in Life Expectancy among Treated HIV-Positive Individuals in the United States and Canada. PLoS One 2013; 8(12): e81355.

10. Guaraldi G, Zona S, Brothers TD, et al. Aging with HIV vs. HIV seroconversion at older age: a diverse population with distinct comorbidity profiles. PLoS One. 2015;10(4):e0118531.

11. CINECA ARNO Observational database. Available at: https://www.cineca.it/en/ content/arno-observatory. Accessed: April 242018.

12. EACS European AIDS Society Guideline. Available at http://www.eacsociety.org/ guidelines/eacs-guidelines/eacs-guidelines.html. Last visit 2nd April 2018.

13. Fortin M, Bravo G, Hudon C, Lapointe L, Almirall J, Dubois MF, Vanasse A: Relationship between multimorbidity and health-related quality of life of patients in primary care. Qual Life Res 2006, 15(1):83-91.

14. Starfield B: Challenges to primary care from co- and multi-morbidity. Primary Health Care Research \& Development 2011; 12: 1-2

15. Hasse B, Ledergerber B, Furrer H, Battegay M, Hirschel B, Cavassini M, Bertisch B, Bernasconi E, Weber R: Morbidity and aging in HIV-infected persons: the Swiss HIV cohort study. Clin Infect Dis. 2011;53(11):1130-9.

16. Hersh BS, Popovici F, Apetrei RC et al. Acquired immunodeficiency syndrome in Romania. Lancet. 1991;338(8768):645-9.

17. Lai A, Bozzi G, Franzetti M, Binda F, Simonetti FR, De Luca A, et al. (2016) HIV-1 A1 Subtype Epidemic in Italy Originated from Africa and Eastern Europe and Shows a High Frequency of Transmission Chains Involving Intravenous Drug Users. PLoS ONE 11(1): e0146097.

18. Tavoschi L, Gomes Dias J, Pharris A; EU/EEA HIV Surveillance Network. New HIV diagnoses among adults aged 50 years or older in 31 European countries, 2004-15: an analysis of surveillance data. Lancet HIV. 2017 Nov;4(11):e514-e521.

19. La Fleur F. Small (2009) What Older Adults Know about HIV/AIDS: Lessons from an HIV/AIDS Education Program, Educational Gerontology, 36:1, 26-45.

20. Chen Y, Bussell A S, Shen Z, et al. Declining Inconsistent Condom Use but Increasing HIV and Syphilis Prevalence Among Older Male Clients of Female Sex Workers. Medicine; Volume 95, Number 22, June 2016. 